\title{
Surface coupling effects on contact mechanics: contact area and interfacial separation between an elastic solid and a hard substrate with randomly rough, self-affine fractal surfaces
}

\author{
M. Feshanjerdi ${ }^{1}$ A. A. Masoudi $^{1}$ M. Khorrami $^{1}$
}

Received: 7 August 2016/ Accepted: 26 November 2016/Published online: 19 December 2016

(c) The Author(s) 2016. This article is published with open access at Springerlink.com

\begin{abstract}
The objective of this study is to investigate both the contact area and the interfacial separation between two surfaces. Both surfaces are considered to be rough, one of them being elastic and the other one hard. The work is based on an extended version of Persson's model of contact mechanics to study the behavior of the contact area, the interfacial separation and the pressure distribution. The results are compared with the case merely the hard substrate is rough. It is seen that introducing a roughness in the elastic surface decreases the real contact, if the surfaces are uncorrelated. A positive (negative) correlation increases (decreases) the real contact. A reverse pattern occurs for the width of the pressure distribution, as well as the interfacial separation (at equal pressures).
\end{abstract}

Keywords Self-affine fractal · Cross-correlation - Surface effects

\section{Introduction}

All of the surfaces occurring in nature and industry are rough, provided they are observed with sufficiently high magnifications (small length scales) [1, 2]. So, for two contacting solid surfaces, microscopically, there are many

A. A. Masoudi

masoudi@alzahra.ac.ir

M. Feshanjerdi

m.feshanjerdi@alzahra.ac.ir

M. Khorrami

mamwad@mailaps.org

1 Department of Physics, Alzahra University, 1993891167 Tehran, Iran non-contact regions (the interfacial separation), and microscopic contact occurs only at a fraction of the macroscopic contact. This fraction of real contact, as well as the interfacial separation, are affected by the roughness of the surfaces, and play important roles in the mechanical properties of the system. The area of real contact characterizes the frictional properties of the contact, as well as the strength of adhesion and the amount of wear [3-5]. Some other phenomena are affected by the interfacial separation, among which are the heat transfer, the contact resistivity, lubrication, and sealing [5-8]. The effect of the surface roughness on the area of real contact has been studied by two classes of analytical models. The first class involves multiasperity contact theories (originally formulated by Greenwood and Williamson (GW) [9-13]), where the contact between the surfaces is modeled as an ensemble of randomly distributed Hertzian contacts between the asperities. The second class is based on Persson's model of contact mechanics [1, 2], where the probability distribution of the contact pressure is shown to be governed by a diffusive process in terms of the magnification at which the interface is observed. Numerical studies [14-17] have shown that, in the case of non-adhesive contacts, when a flat elastic body is brought into contact with a rough surface, the real contact area increases proportional to the applied normal squeezing pressure (applied load). In [18], it has been shown that the GW-type theories predict linearity only for vanishingly small contact areas, corresponding to vanishingly small applied normal squeezing pressures. When the applied normal squeezing pressure is increased, the theoretical predictions rapidly deviate from the asymptotic linearity. This behavior is not seen in Persson's model, which predicts linearity between contact area and applied normal squeezing pressure for real contact values of up to about 15-20 percent of nominal contact area. This is in agreement with some experimental and numerical 
results. Under full contact conditions, Persson's model is exact, but in the case of partial contact, some numerical results [16, 19] regarding non-adhesive contacts between rough surfaces indicate an underestimation of the contact area in Persson's model, while the results of the model still qualitatively agree with numerical calculations [20].

In all of these works, the area of real contact between a smooth elastic solid surface and a hard substrate with randomly rough surface has been studied. As stated earlier, however, there are essentially no surfaces which are smooth on atomic scales. Here, the elastic solid is assumed to have a rough surface as well, and the effect of roughness on the area of real contact and the interfacial separation is studied. The contacts are assumed to be frictionless and non-adhesive, and the roughness of both surfaces is assumed to be random. An extended version of Persson's model of contact mechanics is used to investigate the area of real contact, as well as the interfacial separation between two surfaces.

The outline of the paper is as follows. In Sect. 2, Persson's model of contact area and interfacial surface separation is reviewed. In Sect. 3, an extended version of Persson's model of contact mechanics is used to calculate the contact area and the interfacial surface separation for randomly rough elastic solids and hard substrates with randomly rough surfaces in contact with each other. Numerical results corresponding to the randomly rough self-affine fractal surfaces are presented in Sect. 4. Sections 3 and 4 contain the main results. The novelty, which is described in these sections, is the introduction of a second rough surface and the investigation of the effects of both surfaces being rough and also the effect of their correlation. Section 5 contains the concluding remarks.

\section{The contact area, and the interfacial surface separation}

The main topics of the Persson's model of contact mechanics are reviewed in this section. The model is based on the investigation of the interfacial at different magnifications $\zeta$, $[1,2]$. The system's saturation length is $L$, the length scale is $\lambda$, and the wave numbers $q$ and $q_{L}$ correspond to the length scale and the size of the system, respectively:

$$
\begin{aligned}
q & =\frac{2 \pi}{\lambda}, \\
q_{L} & =\frac{2 \pi}{L}, \\
\zeta & =\frac{L}{\lambda}, \\
& =\frac{q}{q_{L}},
\end{aligned}
$$

The goal is to find the probability distribution $P(\sigma, \zeta)$ of the normal stress $\sigma$ at the interface under the magnification $\zeta$. The equation governing this distribution is shown to be a diffusion equation. For a non-adhesive contact, the boundary condition is [21]

$P(\sigma=0, \zeta)=0$

and for the resulting probability distribution [14, 22]

$P(\sigma, \zeta)=\frac{1}{2(\pi G)^{1 / 2}}\left\{\exp \left[-\frac{(\sigma-p)^{2}}{4 G}\right]-\exp \left[-\frac{(\sigma+p)^{2}}{4 G}\right]\right\}$,

where $p$ is the nominal squeezing pressure and

$$
G(q)=\frac{\pi}{4}\left(\frac{E}{1-v^{2}}\right)^{2} \int_{q_{L}}^{q} \mathrm{~d} q^{\prime} q^{\prime 3} C\left(q^{\prime}\right),
$$

with $E$ and $v$ being the elastic modulus and the Poisson's ratio of the elastic block, respectively. $C(q)$ is the autospectral density function [23] of the hard randomly rough substrate. Denoting the actual (microscopic) and the nominal (macroscopic) contact areas by $A$ and $A_{0}$, respectively, the relative contact area is

$$
\begin{aligned}
\frac{A}{A_{0}} & =\int_{0^{+}}^{\infty} \mathrm{d} \sigma P(\sigma, \zeta), \\
& =: P(q) .
\end{aligned}
$$

So,

$$
\begin{aligned}
\frac{A}{A_{0}} & =\frac{1}{(\pi G)^{1 / 2}} \int_{0}^{p} \mathrm{~d} \sigma \exp \left(-\frac{\sigma^{2}}{4 G}\right) \\
& =\operatorname{erf}\left(\frac{p}{2 G^{1 / 2}}\right)
\end{aligned}
$$

The error function can be approximated by a linear function of its argument, for the case the argument is small. The result is

$$
\frac{A}{A_{0}} \approx \frac{p}{(\pi G)^{1 / 2}}, \quad p \ll G^{1 / 2} .
$$

Regarding the interfacial surface separation, let us consider an elastic block squeezed against a flat hard surface. The separation between the average surface plane of the block and the average surface plane of the substrate is denoted by $\bar{u}$, which is nonnegative. Due to the external pressure $p$ required to produce this separation, an elastic energy is stored in the block. Denoting this by $U_{\mathrm{el}}$, one arrives at

$$
\begin{aligned}
U_{\mathrm{el}}(\bar{u}) & =\int_{\bar{u}}^{\infty} \mathrm{d} u A_{0} p(u), \\
p & =-\frac{1}{A_{0}} \frac{\mathrm{d} U_{\mathrm{el}}}{\mathrm{d} u}
\end{aligned}
$$


When the elastic block is soft and the hard substrate is rough, Persson's model results in the following for the elastic energy [2, 24-26].

$$
\begin{aligned}
& U_{\mathrm{el}} \approx A_{0} E^{*} \gamma \frac{\pi}{2} \int_{q_{L}}^{q_{a}} \mathrm{~d} q q^{2} P(q) C(q), \\
& E^{*}=\frac{E}{\left(1-v^{2}\right)},
\end{aligned}
$$

where $q_{a}$ is the largest surface-roughness wave vector, and $C(q)$ is the auto-spectral density function of the hard randomly rough surface. In cases where the applied normal squeezing pressure $p$ is small, the surface asperities do not fully penetrate the elastic block and only a partial contact is realized. So, the full contribution of the auto-spectral density function is not received by the elastic energy. In (9), this has been addressed through the factor $\gamma P(q)$, where $P(q)$ is the relative contact area for elastic nonadhesive contact and is given by $[1,21]$

$$
\begin{aligned}
& P(q)=\frac{1}{\sqrt{\pi}} \int_{0}^{S(q) p} \mathrm{~d} x \exp \left(-x^{2}\right) . \\
& S(q)=\frac{w(q)}{E^{*}} . \\
& w(q)=\left[\pi \int_{q_{L}}^{q} \mathrm{~d} q^{\prime} q^{\prime 3} C\left(q^{\prime}\right)\right]^{-1 / 2} .
\end{aligned}
$$

$\gamma$ is less than one (but of the order one), when the squeezing pressures are small, and this factor takes into account the fact that the elastic energy stored in the contact region is less than the average elastic energy for full contact [22].

Substituting (9), (10), (11), and (13) in (8), after some calculations, it is shown that for nonadhesive interactions and small applied pressures, the relation between the average interfacial separation $\bar{u}$ and the small applied normal squeezing pressure $p$ is $[22,24]$.

$$
\begin{aligned}
& p \approx \beta E^{*} \exp \left(-\frac{\bar{u}}{u_{0}}\right), \\
& \bar{u} \approx u_{0} \log \left(\frac{\beta E^{*}}{p}\right),
\end{aligned}
$$

where

$$
\begin{aligned}
& u_{0}=\sqrt{\pi} \gamma \int_{q_{L}}^{q_{a}} \mathrm{~d} q q^{2} C(q) w(q) \\
& \beta=\varepsilon \exp \left\{-\frac{\int_{q_{L}}^{q_{a}} \mathrm{~d} q q^{2} C(q) w(q) \log [w(q)]}{\int_{q_{L}}^{q_{a}} \mathrm{~d} q q^{2} C(q) w(q)}\right\}, \\
& \varepsilon=\exp \left[\int_{0}^{\infty} \mathrm{d} x 2 x \log x \exp \left(-x^{2}\right)\right] \\
& \approx 0.7493 .
\end{aligned}
$$

\section{An extension to the case of two randomly rough surfaces in contact with each other}

As stated before, usually both of surfaces which are in contact with each other are rough. Here, Persson's model of contact mechanics is extended to such cases.

\section{The contact area}

The relation between the Fourier transform of the normal stress $\sigma_{z}(\boldsymbol{q})$ and that of the normal displacement field $u_{z}(\boldsymbol{q})$ of the surface of the elastic solid is [26]:

$u_{z}(\boldsymbol{q})=M_{z z}(\boldsymbol{q}) \sigma_{z}(\boldsymbol{q})$,
$M_{z z}(\boldsymbol{q})=-\frac{2\left(1-v^{2}\right)}{E q}$.

When a randomly rough elastic surface is in contact with the randomly rough surface of a hard substrate, Eq. (18) still holds, but now, if the contact between the surfaces is full, the normal displacement field of the elastic solid is equal to the difference of the heights of the surfaces $[27,28]$

$u_{z}(\boldsymbol{q})=h_{2}(\boldsymbol{q})-h_{1}(\boldsymbol{q})$

so that

$h_{2}(\boldsymbol{q})-h_{1}(\boldsymbol{q})=M_{z z}(\boldsymbol{q}) \sigma_{z}(\boldsymbol{q})$

So, the relative contact area and pressure distribution at the interface $(\sigma>0)$ change. The change could be implemented through a substitution of the autocorrelation function $C$ with the autocorrelation corresponding to $\left(h_{2}-h_{1}\right)$, that is $[27,28]$

$C \rightarrow\left(C_{1}+C_{2}-2 \eta \sqrt{C_{1} C_{2}}\right)$,

where $C_{1}(q)$ and $C_{2}(q)$ are the auto-spectral density functions for the rough substrate and the rough elastic block, respectively, and $\eta$ is the coherence function [29, 30]. The surfaces are assumed to be homogeneous and isotropic, so (4) is transformed into:

$$
\begin{aligned}
G(q)= & \frac{\pi}{4}\left(\frac{E}{1-v^{2}}\right)^{2} \int_{q_{L}}^{q} \mathrm{~d} q^{\prime} q^{\prime 3}\left[C_{1}\left(q^{\prime}\right)+C_{2}\left(q^{\prime}\right)\right. \\
& \left.-2 \eta \sqrt{C_{1}\left(q^{\prime}\right) C_{2}\left(q^{\prime}\right)}\right] .
\end{aligned}
$$

This new form of $G$ is to be put in (6) and (7), to obtain the contact area. Here, $\eta$ is considered to be a constant (independent of $\boldsymbol{q}$ ), and the results for this simple case are presented. Special cases are $\eta=0$ (uncorrelated surfaces), $\eta=+1$ (completely positive correlated surfaces), or $\eta=$ -1 (completely negative correlated surfaces). 


\section{The interfacial surface separation}

When a randomly rough elastic solid is squeezed against a randomly rough surface of a hard substrate, the elastic energy is similar to (9), but with $C$ substituted according to (22) $[27,28]$ :

$$
\begin{aligned}
U_{\mathrm{el}} \approx & \frac{\pi E A_{0} \gamma}{2\left(1-v^{2}\right)} \int_{q_{L}}^{q_{a}} \mathrm{~d} q q^{2} P(q)\left[C_{1}(q)+C_{2}(q)\right. \\
& \left.-2 \eta \sqrt{C_{1}(q) C_{2}(q)}\right] .
\end{aligned}
$$

The relative contact area for elastic nonadhesive contact is obtained from (11) with

$$
w(q)=\left(\pi \int_{q_{L}}^{q} \mathrm{~d} q^{\prime} q^{\prime 3}\left[C_{1}\left(q^{\prime}\right)+C_{2}\left(q^{\prime}\right)-2 \eta \sqrt{C_{1}\left(q^{\prime}\right) C_{2}\left(q^{\prime}\right)}\right]\right)^{-1 / 2} .
$$

Using (11),

$$
\frac{\partial P}{\partial \bar{u}}=\frac{2}{\sqrt{\pi}} S(q) \exp \left\{-[S(q)]^{2} p^{2}\right\} \frac{\mathrm{d} p}{\mathrm{~d} \bar{u}} .
$$

Substituting (24) and (26) in (8) results in:

$$
\begin{aligned}
\mathrm{d} \bar{u}= & -\sqrt{\pi} \gamma \int_{q_{L}}^{q_{a}} \mathrm{~d} q q^{2}\left[C_{1}(q)+C_{2}(q)-2 \eta \sqrt{C_{1}(q) C_{2}(q)}\right] \\
& \times w(q) \exp \left\{-\left[\frac{w(q) p}{E^{*}}\right]^{2}\right\} \frac{\mathrm{d} p}{p} .
\end{aligned}
$$

Integrating this from $\bar{u}=0$ (full contact, corresponding to $p=\infty)$ to $\bar{u}$ gives:

$$
\begin{aligned}
\bar{u}= & \sqrt{\pi} \gamma \int_{q_{L}}^{q_{a}} \mathrm{~d} q q^{2}\left[C_{1}(q)+C_{2}(q)-2 \eta \sqrt{C_{1}(q) C_{2}(q)}\right] \\
& \times w(q) \int_{p}^{\infty} \frac{\mathrm{d} p^{\prime}}{p^{\prime}} \exp \left\{-\left[\frac{w(q) p^{\prime}}{E^{*}}\right]^{2}\right\} .
\end{aligned}
$$

For very low squeezing pressures, one has

$$
\begin{aligned}
& p \approx \beta E^{*} \exp \left(-\frac{\bar{u}}{u_{0}}\right) \\
& u_{0}=\sqrt{\pi} \gamma \int_{q_{L}}^{q_{a}} \mathrm{~d} q q^{2}\left[C_{1}(q)+C_{2}(q)-2 \eta \sqrt{C_{1}(q) C_{2}(q)}\right] w(q),
\end{aligned}
$$

$$
\begin{aligned}
& \beta=\varepsilon \exp \\
& \left\{-\frac{\int_{q_{L}}^{q_{a}} \mathrm{~d} q q^{2}\left[C_{1}(q)+C_{2}(q)-2 \eta \sqrt{C_{1}(q) C_{2}(q)}\right] w(q) \log [w(q)]}{\int_{q_{L}}^{q_{a}} \mathrm{~d} q q^{2}\left[C_{1}(q)+C_{2}(q)-2 \eta \sqrt{C_{1}(q) C_{2}(q)}\right] w(q)}\right\},
\end{aligned}
$$

with $\varepsilon$ being obtained from (17).

\section{Numerical results}

In all cases, it is assumed that the randomly rough elastic solid surface and the randomly rough surface of the hard substrate are both self-affine fractal surfaces with the autospectral density functions described by [24]:

$C(q) \approx \frac{H}{\pi}\left(\frac{h_{\mathrm{rms}}}{q_{L}}\right)^{2}\left(\frac{q}{q_{L}}\right)^{-(2 H+2)}$,

where $H$ is the Hurst exponent, and it is assumed that $q_{a}$ is much larger than $q_{L}$, which usually is. For the hard substrate and the elastic block, these values have been used.

$$
\begin{aligned}
& q_{L}=2 \times 10^{8} \mathrm{~m}^{-1}, \quad q_{a}=4 \times 10^{10} \mathrm{~m}^{-1}, \\
& H_{1}=0.8, \quad h_{\mathrm{rms} 1}=1 \mathrm{~nm}, \\
& H_{2}=0.7, \quad h_{\mathrm{rms} 2}=1 \mathrm{~nm}, \\
& E=77.2 \mathrm{GPa}, \quad v=0.42 .
\end{aligned}
$$

\section{Contact area}

Figure 1 shows the contact area ratio $A / A_{0}$ calculated from (6) and (23), as a function of the normalized pressure $p / E^{*}$ for the magnification $\zeta=4$.

The pressure distribution calculated from (3) and (23), as a function of the normalized pressure $\sigma / E^{*}$, is shown in Fig. 2 for $\zeta=4$ and for three different nominal pressures.

In these curves, the circle curve is for the case where only the substrate is rough. The asterisk, solid, and dotted curves correspond to the cases where both surfaces are rough and uncorrelated $(\eta=0)$, completely positively correlated $(\eta=1)$, and completely negatively correlated

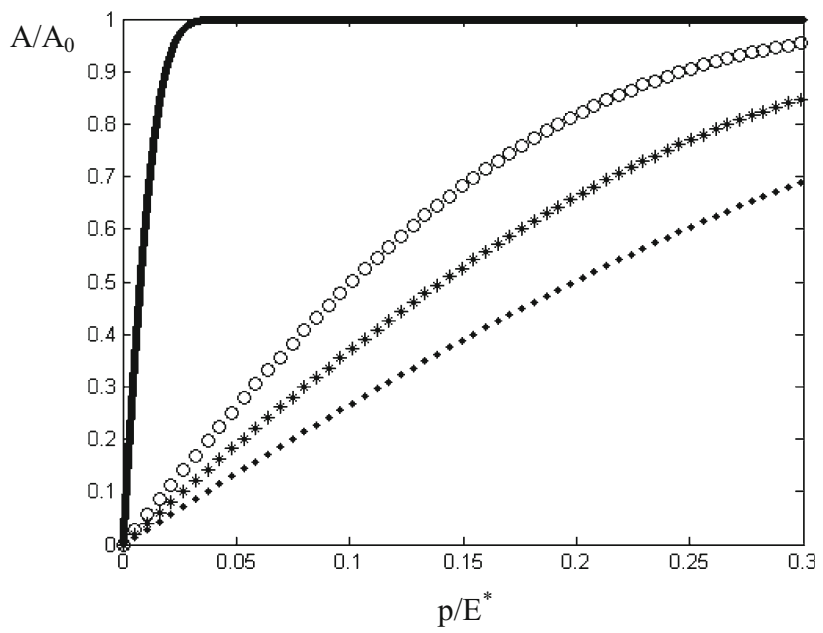

Fig. 1 The contact area ratio $A / A_{0}$ as a function of the normalized pressure $p / E^{*}$ for the magnification $\zeta=4$. The circle curve is for the case where only the substrate is rough. The asterisk, solid, and dotted curves correspond to the cases where both surfaces are rough and uncorrelated $(\eta=0)$, completely positively correlated $(\eta=1)$, and completely negatively correlated $(\eta=-1)$, respectively 

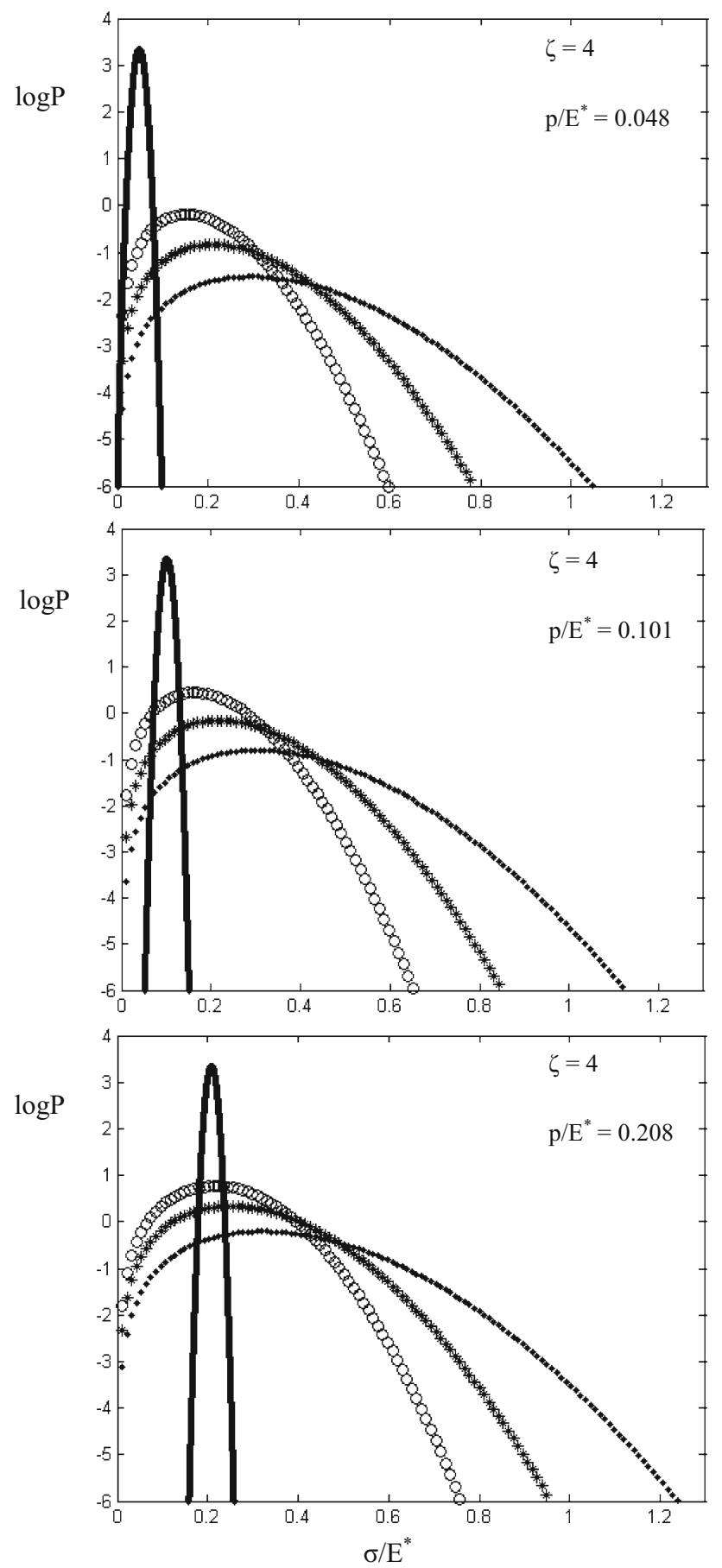

Fig. 2 The pressure distribution as a function of the normalized pressure $\sigma / E^{*}$ for the magnification $\zeta=4$ and for three different nominal pressures. The circle curve is for the case where only the substrate is rough. The asterisk, solid, and dotted curves correspond to the case where both surfaces are rough and uncorrelated $(\eta=0)$, completely positively correlated $(\eta=1)$, and completely negatively correlated $(\eta=-1)$, respectively

$(\eta=-1)$, respectively. Figure 2 shows that both surfaces being rough, but uncorrelated, results in a decrease in the contact area, compared to the case of only one rough surface. If both surfaces are rough, and they are correlated,

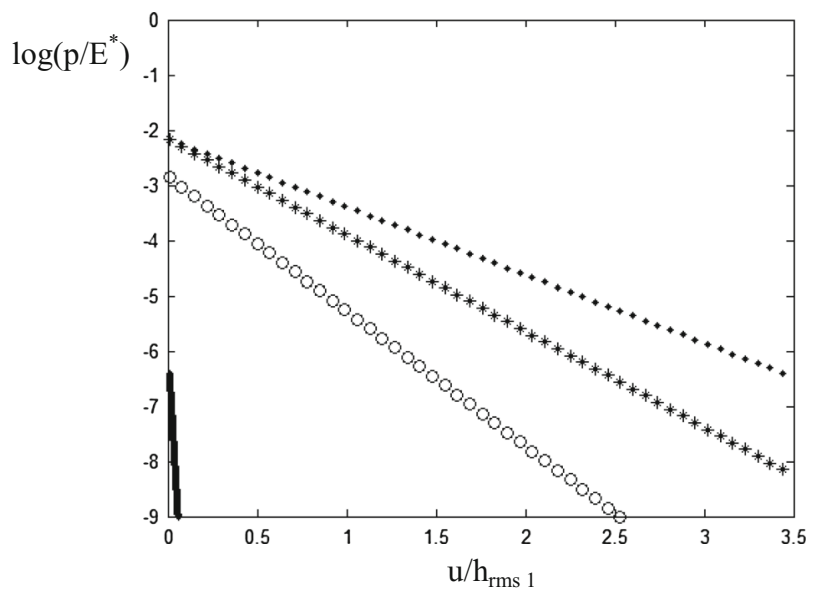

Fig. 3 The logarithm of the normalized average pressure $p / E^{*}$, as a function of the separation $\bar{u}$ between the average plane of the substrate and the average plane of the lower surface of the elastic block for the magnification $\zeta=4$. The circle curve is for the case where only the substrate is rough. The asterisk, solid, and dotted curves correspond to the case where both surfaces are rough and uncorrelated $(\eta=0)$, completely positively correlated $(\eta=1)$, and completely negatively correlated $(\eta=-1)$, respectively

depending on the sign of the correlation, an increase or decrease in the values of the contact area is resulted, compared to the case of two uncorrelated surfaces and the case of only one rough surface. A positive correlation $(\eta=1)$ increases the contact area between the two surfaces, so that the pressure distribution vanishes in a smaller normalized pressure, as seen from Fig. 2. For a negative correlation $\eta=-1$, however, the contact area is decreased compared to the case of uncorrelated surfaces and the case of only rough surface, so that the pressure distribution vanishes in larger pressure. It is seen that when both surfaces are rough but uncorrelated, the width of the pressure distribution is larger compared to the case of only one rough surface. A positive (negative) correlation results in a decrease (an increase) of the width of the pressure distribution.

\section{Interfacial surface separation}

The logarithm of the normalized average pressure $p / E^{*}$, as a function of the separation $\bar{u}$ between the average plane of the substrate and the average plane of the lower surface of the elastic block is shown in Figs. 3 and 4. Figure 3 corresponds to the magnification $\zeta=4$, and Fig. 4 corresponds to the magnification $\zeta=200$. In these curves, the circle curve is for the case where only the substrate is rough. The asterisk, solid, and dotted curves correspond to the cases where both surfaces are rough and uncorrelated $(\eta=0)$, completely positively correlated $(\eta=1)$, and completely negatively correlated $(\eta=-1)$, respectively. It is seen that the interfacial separation at a fixed pressure is larger when 


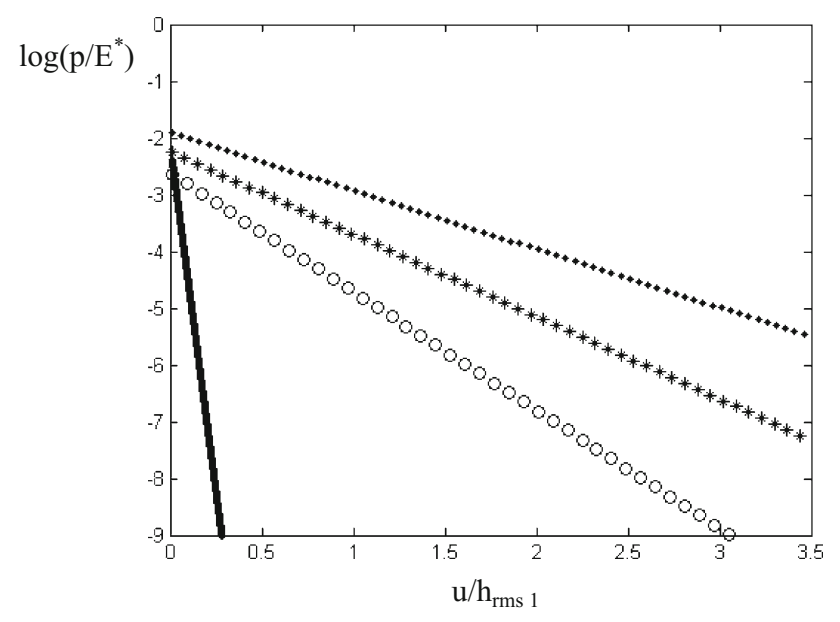

Fig. 4 The logarithm of the normalized average pressure $p / E^{*}$, as a function of the separation $\bar{u}$ between the average plane of the substrate and the average plane of the lower surface of the elastic block for the magnification $\zeta=200$. The circle curve is for the case where only the substrate is rough. The asterisk, solid, and dotted curves correspond to the cases where both surfaces are rough and uncorrelated $(\eta=0)$, completely positively correlated $(\eta=1)$, and completely negatively correlated $(\eta=-1)$, respectively

both surfaces are rough but uncorrelated. A positive (negative) correlation decreases (increases) the interfacial separation.

\section{Concluding remarks}

An extension of Persson's model of contact mechanics was used to study the contact area and the interfacial separation, when the elastic solid and the hard substrate are both rough. It was seen that when the two surfaces are rough but uncorrelated, the real contact decreases compared to the case where only the substrate is rough, effectively the roughness has been increased. It was also shown that when the surfaces are correlated, a positive correlation increases the real contact area compared to the case of no correlation, while a negative correlation decreases the real contact area compared to the case of no correlation. A reverse pattern is seen for the width of the pressure distribution, as well as the interfacial separation (at equal pressures): making both surfaces rough but uncorrelated increases these, and a positive (negative) correlation results in a decrease (an increase) in these.

Acknowledgements This work was supported by the research council of the Alzahra University.

Open Access This article is distributed under the terms of the Creative Commons Attribution 4.0 International License (http://crea tivecommons.org/licenses/by/4.0/), which permits unrestricted use, distribution, and reproduction in any medium, provided you give appropriate credit to the original author(s) and the source, provide a link to the Creative Commons license, and indicate if changes were made.

\section{References}

1. Persson, B.N.J.: Theory of rubber friction and contact mechanics. J. Chem. Phys. 115, 3840 (2001)

2. Persson, B.N.J.: Adhesion between an elastic body and a randomly rough hard surface. Eur. Phys. J. E. 8, 385 (2002)

3. Bowden, F.P., Tabor, D.: Friction and Lubrication of Solids. Wiley, New York (1956)

4. Johnson, K.L.: Contact Mechanics. Cambridge University Press, Cambridge (1985)

5. Persson, B.N.J.: Sliding Friction: Physical Principles and Applications, 2nd edn. Springer, Berlin (2000)

6. Volokitin, A.I., Persson, B.N.J.: Near-field radiative heat transfer and noncontact friction. Rev. Mod. Phys. 79, 1291 (2007)

7. Rabinowicz, E.: Friction and Wear of Materials, 2nd edn. Wiley, New York (1995)

8. Patir, N., Cheng, H.S.: An average flow model for determining effects of three dimensional roughness onpartial hydrodynamic lubrication. J. Lubr. Technol. 100, 12 (1978)

9. Greenwood, J.A., Williamson, J.B.P.: Contact of nominally flat surfaces. Proc. R. Soc. Lond. A 295, 300 (1966)

10. Bush, A.W., Gibson, R.D., Thomas, T.R.: The elastic contact of a rough surface. Wear 35, 87 (1975)

11. Thomas, T.R.: Rough Surfaces, vol. 8. Longman Group Limited, London (1982)

12. Greenwood, J.A.: A simplified elliptic model of rough surface contact. Wear 261, 191 (2006)

13. Carbone, G.: A slightly corrected Greenwood and Williamson model predicts asymptotic linearity between contact area and load. J. Mech. Phys. Solids 57, 1093 (2009)

14. Yang, C., Tartaglino, U., Persson, B.N.J.: A multiscale molecular dynamics approach to contact mechanics. Eur. Phys. J. E 19, 47 (2006)

15. Borri-Brunetto, M., Chiaia, B., Ciavarella, M.: Incipient sliding of rough surfaces in contact: a multiscale numerical analysis. Comput. Methods Appl. Mech. Eng. 190, 6053 (2001)

16. Hyun, S., Pei, L., Molinari, J.F., Robbins, M.O.: Finite-element analysis of contact between elastic self-affine surfaces. Phys. Rev. E 70, 026117 (2004)

17. Campana, C.: Using Green's function molecular dynamics to rationalize the success of asperity models when describing the contact between self-affine surfaces. Phys. Rev. E 78, 026110 (2008)

18. Carbone, G., Bottiglione, F.: Asperity contact theories: do they predict linearity between contact area and load?. J. Mech. Phys. Solids 56, 2555 (2008)

19. Yang, C., Persson, B.N.J.: Molecular dynamics study of contact mechanics: contact area and interfacial separation from small to full contact. Phys. Rev. Lett. 100, 024303 (2008)

20. Campana, C., Mü̈er, M.H., Robbins, M.O.: Elastic contact between self-affine surfaces: comparison of numerical stress and contact correlation fucntions with analytic predictions. J. Phys.: Condens. Mater. 20, 354013 (2008)

21. Persson, B.N.J., Bucher, F., Chiaia, B.: Elastic contact between randomly rough surfaces: comparison of theory with numerical results. Phys. Rev. B 65, 184106 (2002)

22. Yang, C., Persson, B.N.J.: Contact mechanics: contact area and interfacial separation from small contact to full contact. J. Phys.: Condens. Matter. 20, 215214 (2008)

23. Persson, B.N.J., Albohr, O., Tartaglino, U., Volokitin, A.I., Tosatti, E.: On the nature of surface roughness with application to 
contact mechanics, sealing, rubber friction and adhesion. J. Phys.: Condens. Matter. 17, R1 (2005)

24. Persson, B.N.J.: Relation between interfacial separation and load: a general theory of contact mechanics. Phys. Rev. Lett. 99, 125502 (2007)

25. Persson, B.N.J.: Contact mechanics for randomly rough surfaces. Surf. Sci. Rep. 61, 201 (2006)

26. Persson, B.N.J., Tosatti, E.: The effect of surface roughness on the adhesion of elastic solids. J. Chem. Phys. 115, 5597 (2001)

27. Feshanjerdi, M., Masoudi, A.A., Khorrami, M.: Surface coupling effects on the adhesive contact between an elastic solid and a hard substrate with randomly rough, self-affine fractal surfaces. J. Stat. Mech. 2015(2), P02018 (2015)

28. Feshanjerdi, M., Khorrami, M., Masoudi, A.A.: The hysteretic contribution of friction for the polished rubber on the concrete surface. Appl. Surf. Sci 394, 528 (2017)

29. Bendat, J.S., Piersol, A.G.: Random Data: Analysis and Measurement Procedures, 3rd edn. Wiley, NewYork (2000)

30. Palasantzas, G., Barnas, J., Hosson, J.T.M.D.: Correlated roughness effects on electrical conductivity of quantum wires. J. Appl. Phys. 89, 8002 (2001) 